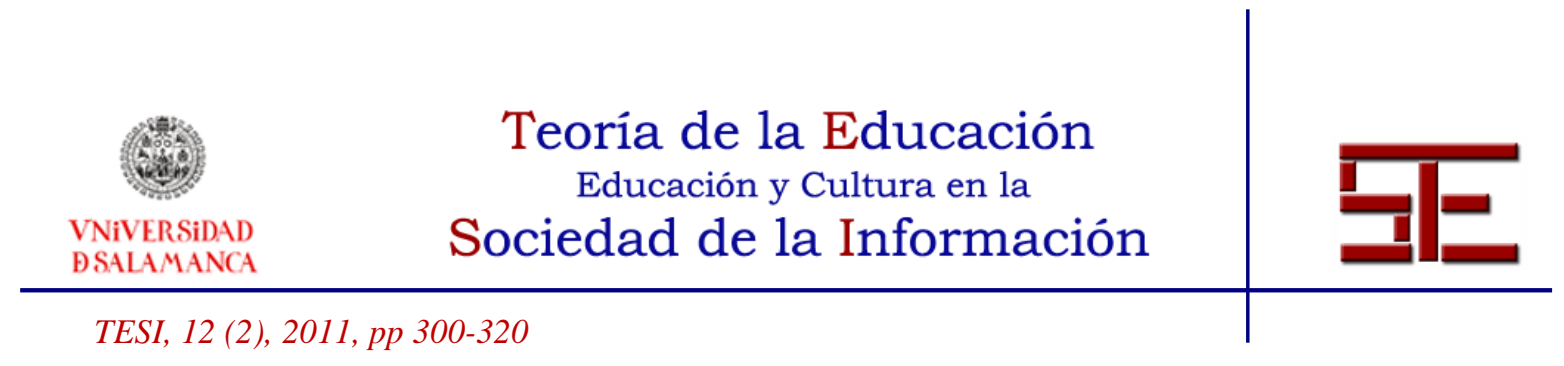

\title{
EXPERIENCIAS COEDUCATIVAS DEL PROFESORADO DE EDUCACIÓN FÍSICA Y RELACIÓN CON LOS CONTENIDOS DE LA MATERIA
}

Resumen: Los objetivos de este estudio fueron conocer las vivencias coeducativas del profesorado en relación con los contenidos de Educación Física y analizar las diferentes estrategias metodológicas que se utilizan en cada bloque de contenidos. Se realizaron entrevistas semiestructuradas sometidas a análisis de contenido. Mediante el proceso hermenéutico se destaca que: (I) la categoría "Bloque de contenidos de Condición Física y Salud" demuestra que el profesorado pretende que las diferencias fisiológicas por cuestión de sexo no se reproduzcan; (II) la categoría "Bloque de contenidos de Expresión Corporal" señala que existe profesorado que no aborda estos contenidos porque los desconoce; (III) la categoría "Bloque de contenidos de Juegos y Deportes" resalta que el principal problema que encuentra el profesorado son los estereotipos que ciertos deportes llevan asociados; la categoría (IV) "Bloque de contenidos de Actividades en el Medio Natural" pone de manifiesto que ninguno de los dos sexos dificulta su trabajo.

Palabras clave: Coeducación; Educación Física; Contenidos: Profesorado.

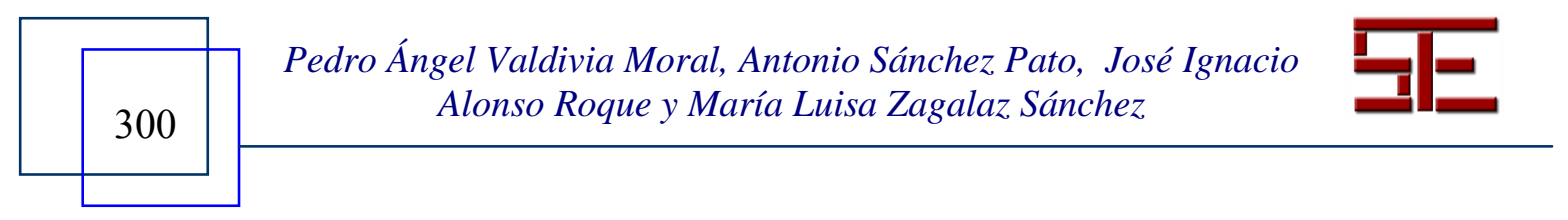




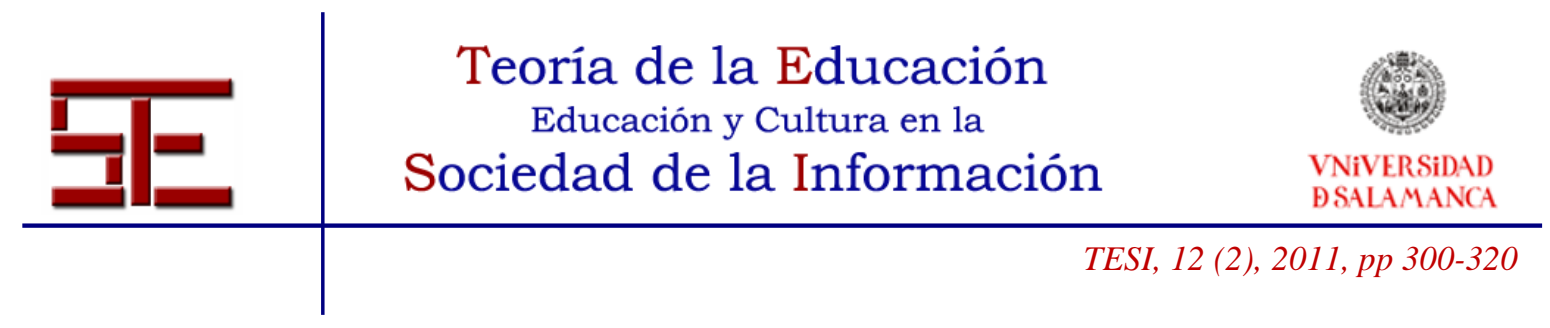

\title{
CO-EDUCATIONAL EXPERIENCES OF PHYSICAL EDUCATION TEACHERS IN RELATION WITH THE CONTENTS OF THE SUBJECT
}

\begin{abstract}
The aims of this study were to be familiar with the co-educational experiences of teachers in relation to the contents of Physical Education and to analyze the different methodological strategies which are used in each block of content. Semi structured interviews were realized subjected to content's analysis. Through the hermeneutic process it is emphasized that: (I) the category "Content's Block of Fitness and Health" shows that the teacher pretends that the physiological differences that are produced by matter of sex don't happen again; (II) the category "Content's Physical Language" notes that there are teachers who don't deal with these contents because they don't recognize them; (III) the category "Content's Block Games and Sports" underlines that the main problem that the teachers find is the stereotypes that some sports take associated; the category (IV) "Content's Block of Activities in the Natural Environment" declares that neither of the two sexes difficult its work.
\end{abstract}

Keywords: Coeducation; Physical Education; Content; Teachers.

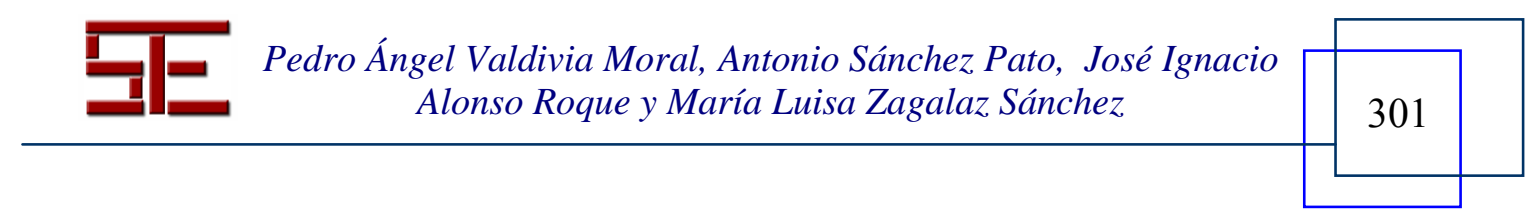




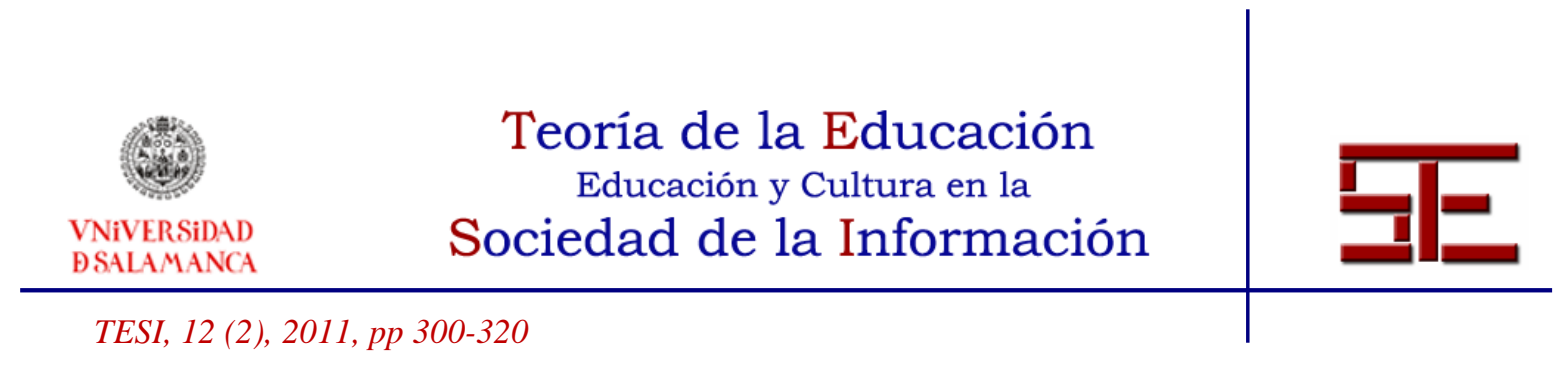

\section{EXPERIENCIAS COEDUCATIVAS DEL PROFESORADO DE EDUCACIÓN FÍSICA Y RELACIÓN CON LOS CONTENIDOS DE LA MATERIA}

Fecha de recepción: 30/04/2010; fecha de aceptación: 12/07/2011; fecha de publicación: 27/07/2011

Pedro Ángel Valdivia Moral

pvaldiviamoral@hotmail.com

Grupo de Investigación HUM 653

Antonio Sánchez Pato

apato@pdi.ucam.edu

Universidad Católica San Antonio de Murcia

José Ignacio Alonso Roque

jialonso@um.es

Universidad de Murcia

María Luisa Zagalaz Sánchez

1zagalaz@ujaen.es

Universidad de Jaén

\section{INTRODUCCIÓN}

En las últimas décadas la mujer ha alcanzado un mayor grado de consideración social, fruto de un cambio de actitudes y comportamientos (Vázquez y Alfaro, 2006). El sistema educativo español es sensible a estos cambios, habiendo realizado numerosas reformas desde los órganos de gobierno (Tainta, 2007). Los cambios más destacados son: A) La Ley General de Educación de 1970, que instituye la escuela mixta en los centros públicos y concertados (Jiménez, Ramos y Cervelló, 2002). B) La Ley Orgánica de Ordenación General del Sistema Educativo (LOGSE) de 1990, que, entre sus aspectos más relevantes, destaca la igualdad de oportunidades entre hombres y mujeres y la no discriminación en la educación por razones de sexo. C) En 2006 la Ley Orgánica de Educación (LOE), que contiene entre sus principios la equidad, la igualdad de oportunidades y la no discriminación.

A pesar de la evolución acarreada por las reformas educativas la escuela mixta no ha conseguido, de hecho, la igualdad entre hombres y mujeres, haciéndose patente la necesidad de realizar cambios más profundos para alcanzar los objetivos coeducativos

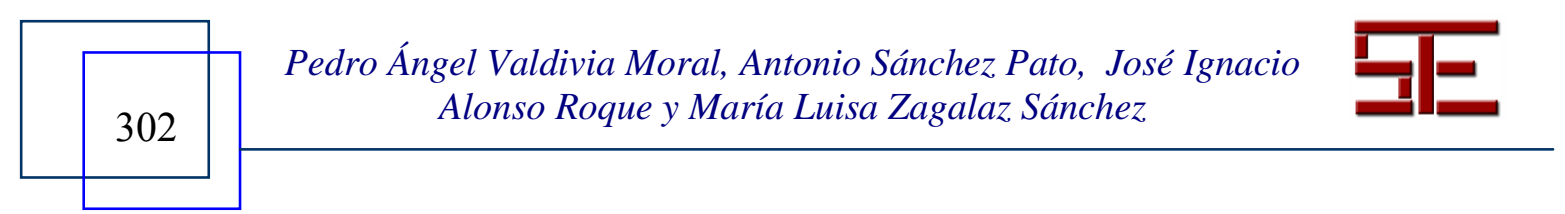




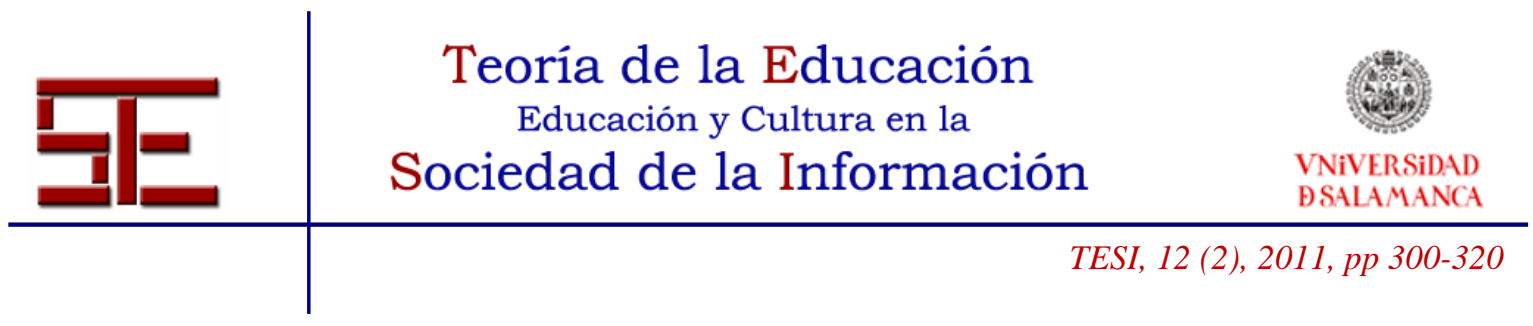

(Subirats, 1994; González, 2005). Se hace necesaria una reflexión por parte del profesorado sobre la situación de los sexos en el aula, con el objetivo de conocer las necesidades del alumnado, para optar por actuaciones lo más equitativas posibles (Freixas, García, Jiménez, Sánchez y Santos, 1993). En este sentido, cabe destacar que Salomone (2009) propone que se realice un seguimiento de las diferencias entre los sexos a medida que éstos se desarrollan, considerando a su vez las diferencias existentes en el alumnado del mismo sexo.

A lo largo de este texto utilizaremos indistintamente el término "sexo", cuando nos refiramos a características biológicas. Igualmente, recurriremos al término "género", cuando hagamos referencia a la forma en la que las diferencias biológicas se interpretan en comportamientos, valores, expectativas sociales, etc., propias de cada uno de los sexos (Freixas et al., 1993; Mosquera y Puig, 1998; Pérez y Carbó, 2010).

Existen diferentes enfoques sobre coeducación que aportan diferentes matices en función de áreas como la pedagogía, la psicología, la sociología, etc. (Valdivia, Sánchez, Alonso y Zagalaz, 2010). En este estudio utilizaremos las concepciones aportadas desde la Educación Física (EF), donde se destacan los siguientes enfoques: a) promover la igualdad de trato entre sexos, no aceptando el modelo universal masculino y desarrollando las cualidades de cada individuo independientemente (Zagalaz et al., 2000); b) asegurar un trato equitativo para chicas y chicos, superando los estereotipos de género, al llevar a la práctica cada una de las actividades que se desarrollan en el contexto educativo (Fontecha, 2006); c) enseñar a valorar tanto a chicos como a chicas los rasgos de carácter positivo de los modelos femenino y masculino, entendiendo que no se deben eliminar formas de comportamiento, sino hacer participar a los chicos de las ventajas que contienen las formas de conducta propias de las mujeres y viceversa (Martínez-Galindo, 2006).

El problema con la EF y su relación con el género reside en la visión de considerar a las niñas un problema desde el punto de vista del rendimiento. La escuela y la EF deben luchar contra los estereotipos de género que se transmiten y que tratan aspectos tales como las pautas de comportamiento o los cánones de belleza (Azzarito y Solmon, 2009).

El periodo escolar es el periodo propicio para desarrollar los factores educativos, si bien no se debe trabajar la coeducación aisladamente como se hace, por ejemplo, en lengua o biología. Esto supondría distorsionar y empobrecer elementos de la educación

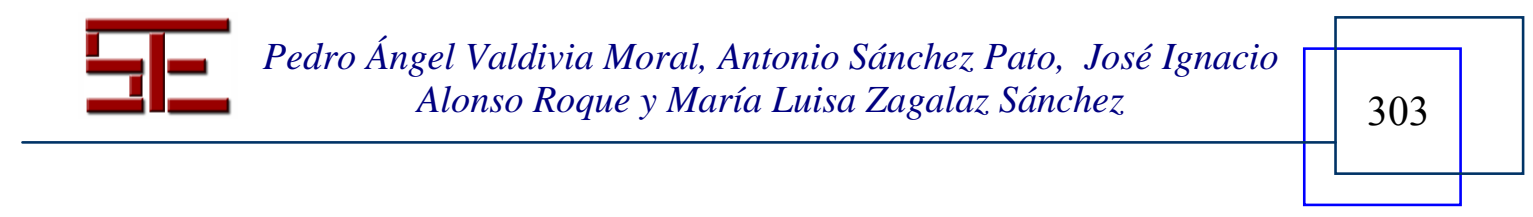




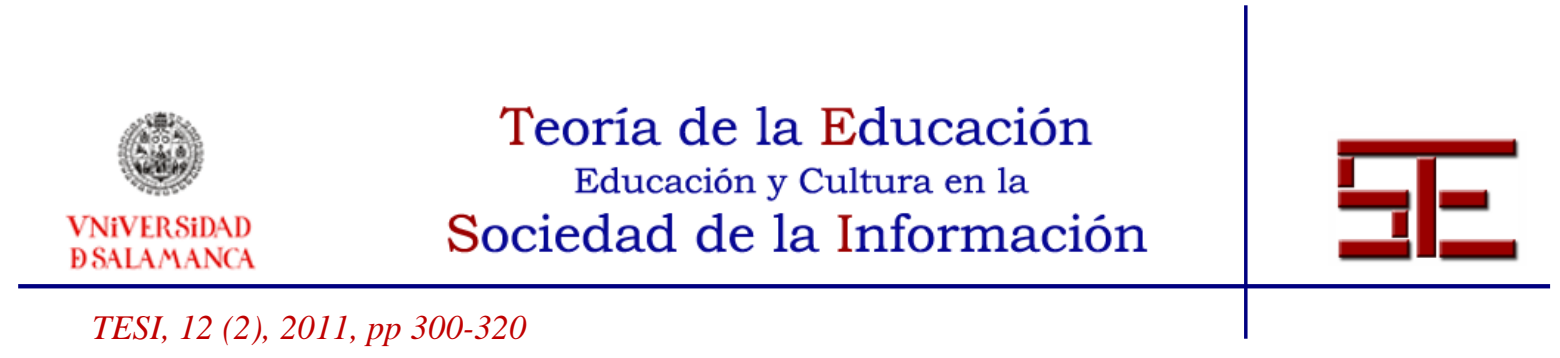

que tienen que ver con actitudes, relaciones, expectativas y valores (Freixas et al., 1993). Es decir, la coeducación es parte del currículum educativo común a todas las áreas y tiene como objetivo que tanto alumnos como alumnas adquieran unos valores que les permitan convivir en sociedad (Martínez-Quesada, 2009). Esto exige la utilización de estrategias educativas concretadas en pautas metodológicas que incluyan la reflexión de la práctica, tal y como proponen Mosquera, Sánchez y Bada (2004).

La escuela mixta no desarrolla la coeducación, aunque conlleva un cierto trato de igualdad entre el alumnado (Castillo, Martínez-López y Zagalaz, 2010; Subirats y Brullet, 1988; Subirats, 1994; Vázquez, 1990). En esta línea, Valdivia (2010) indica que la reunión de chicos y chicas bajo el mismo currículum y en la misma aula no conlleva la solución de la discriminación por sexo. Las diferencias se acentúan en la clase de EF, donde Fasting (1989) señala que:

1. En los juegos y deportes de equipo o colectivos los chicos acaparan las actividades.

2. Las chicas asumen papeles subordinados, siendo los chicos los líderes.

3. En situaciones mixtas las chicas están menos implicadas motrizmente y, por lo tanto, obtienen perores resultados que los chicos.

Respecto a la comunicación que tiene el alumnado con el profesor, Nicaise, Cogerino, Faiclough, Bois y Davis (2007) indican que en clases de bádminton las chicas reciben más interacción con el docente, mientras que en clases de entrenamiento con pesas son los chicos los que la reciben. Por otro, Derry y Allen (2004, citados en Koca, 2009) indican que las chicas tienen más interacción con sus docentes en clases segregadas.

La EF debe formar personas que valoren y respeten los valores sociales indistintamente del sexo. Por lo tanto, deben proporcionar experiencias positivas para que el alumnado mantenga la práctica de actividad física (Valdivia, 2010). La actual orientación de los programas de actividad física a nivel internacional está enfocada hacía el desarrollo de actividad física para chicas adolescentes, donde la EF debe impulsar la implementación de estos programas y combatir las desigualdades de género (Fernández et al., 2006).

Los datos de Moreno, Alonso, Martínez-Galindo y Cervelló (2005) ponen de manifiesto que el alumnado que tiene mayor preferencia por la $\mathrm{EF}$ es aquel que percibe

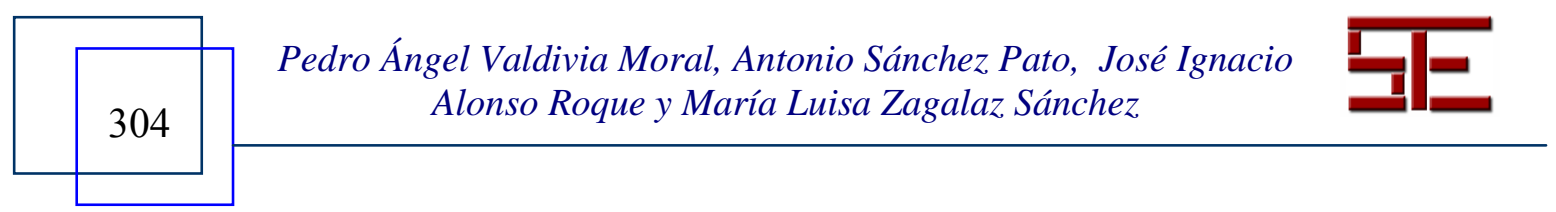




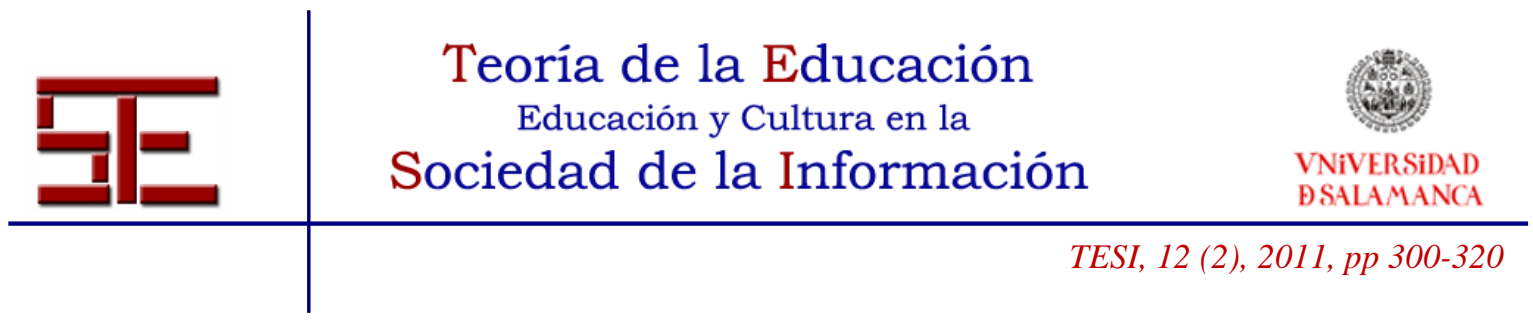

una mayor igualdad de trato y tiene mayor predisposición hacia las actividades. Los datos corroboran que la mayoría de mujeres adultas que realizan actividad física se han iniciado en edad escolar, siendo la adolescencia la etapa con mayor nivel de abandono (Vázquez y Alfaro, 2006). Fernández et al. (2006) señalan los cursos de secundaria como etapa clave, ya que es donde se producen los principales cambios corporales.

El alumnado, además de diferenciarse por el sexo también lo hace por ideologías y actitudes; dentro de la clase de EF se diferencian por el nivel de habilidad. Por lo tanto, es necesario que el profesorado esté sensibilizado ante estas características para evitar prejuicios (Freixas et al., 1993). Por esta razón, Fernández et al. (2006) proponen realizar una revisión crítica del currículum de EF de forma que se contemplen las características del alumnado mediante una exploración e indagación de las posibilidades que ofrece la EF.

Respecto al profesorado, cabe destacar que el 50\% de los estudiantes de la licenciatura en Ciencias de la Actividad Física y del Deporte (CAFD) se especializan en la docencia (Gallo, González y Salinero, 2010), aunque esto no conlleva una formación especifica en coeducación. Aún así, Gray y Wilson (2006) indican en su estudio que el $71 \%$ de los docentes encuestados prefieren dar clase en aulas coeducativas en lugar de aquellas de un solo género. Además, el $52 \%$ de la muestra encuentra las clases coeducativas como menos estresantes.

\subsection{Relación de los contenidos curriculares de Educación Física con la coeducación}

Para la realización de este estudio se han seguido la LOE (2006) y el RD 1631/2006, donde vienen descritos los contenidos específicos para EF en secundaria en España a pesar de que existen comunidades autónomas que desarrollan su propio currículum. En la LOGSE (1990) se contemplaba la coeducación como contenido transversal, sin embargo, actualmente se considera como parte de la educación en valores que el alumnado debe adquirir tal y como se contempla en la Ley de Educación de Andalucía (2007), por ejemplo.

La cultura es el punto de origen y referencia de donde se diseñan los contenidos que se plasman en el currículum (Ramos y Del Villar, 1999). Por otro lado, debe ser una declaración de intenciones por parte del docente, realizando las adaptaciones necesarias para lograr los fines educativos (Gómez et al., 2008). Por lo tanto, es una herramienta abierta y flexible, siendo el profesorado en última instancia el que ajuste dichos

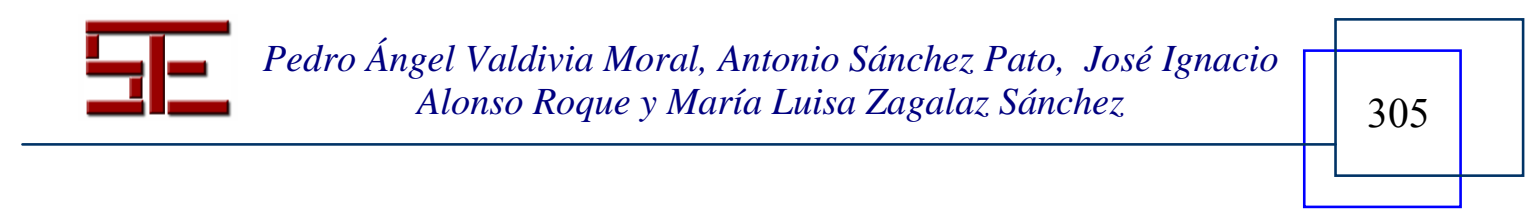




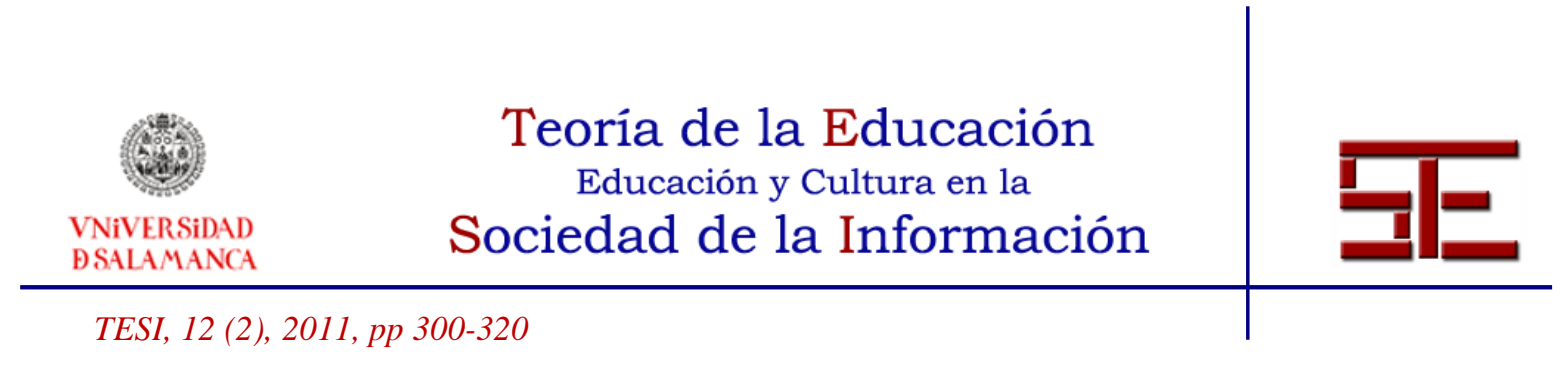

contenidos a sus objetivos, experiencia, alumnado, afinidades del profesorado, contexto social y económico, formación, materiales disponibles, climatología, instalaciones y técnicas y estilos de enseñanza influyentes (Ramos y Del Villar, 1999; Gómez et al., 2008).

Existen contenidos en EF que transmiten una serie de estereotipos que son valorados de forma distinta. Soler (2006) indica que el fútbol está considerado una actividad masculina y tiene una consideración social más alta que, por ejemplo, saltar a la cuerda, que es una actividad asociada al género femenino. González (2005) asocia los contenidos de "Condición Física y Salud" y "Juegos y Deportes" al sexo masculino, vinculando la "Expresión Corporal" al sexo femenino. Dentro del bloque de contenidos de "Juegos y Deportes", Robles, Giménez y Abad (2010) indican que existe una inclinación favorable dentro de las alumnas hacia la práctica de deportes individuales. Mientras tanto, los chicos prefieren los deportes de equipo.

Según González (2005), uno de los aspectos importantes a la hora de la elección de contenidos a impartir durante el curso son los libros de texto, siendo una fuente de sexismo. La investigación de González (2005) pone de manifiesto que todos los libros de texto de EF tienen un mayor contenido del bloque de "Condición Física y Salud" y/o "Juegos y Deportes", no superando en ningún caso la "Expresión Corporal" en número de páginas a los demás contenidos. Dentro del bloque de contenidos de "Juegos y Deportes" se presta más atención a los deportes colectivos, tradicionalmente asociados más al género masculino, que a los deportes individuales artísticos.

Dadas las características cualitativas del estudio que hemos llevado a cabo, se hace necesaria una descripción del mismo para analizar los discursos del profesorado entrevistado, a pesar de no encontrar estudios previos que relacionen la coeducación con el currículum de EF. Siguiendo el planteamiento de Zagalaz (2001), en esta investigación se han tenido en cuenta los tres elementos básicos de la enseñanzaaprendizaje: el profesorado, el alumnado y el proceso. A su vez, se incide en las experiencias del profesorado y cómo han repercutido en su práctica diaria respecto al proceso de enseñanza-aprendizaje y al alumnado.

Ante la falta de respuestas concluyentes sobre la materia y la importancia de este factor, planteamos el siguiente problema de investigación, ¿Cómo afecta la coeducación al trabajo de los contenidos por parte del profesorado de EF?

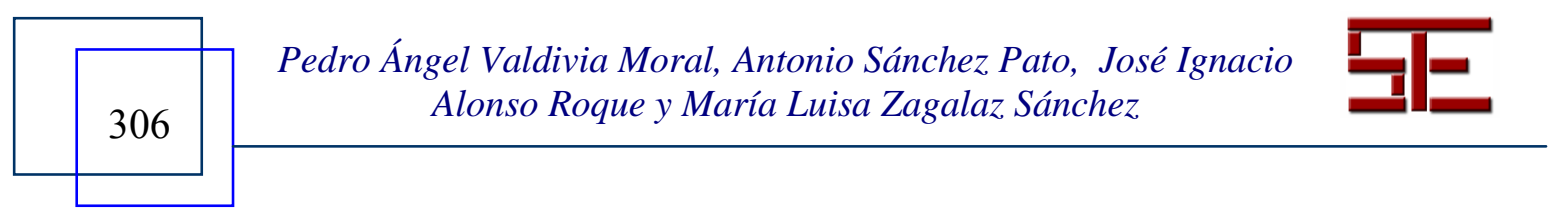




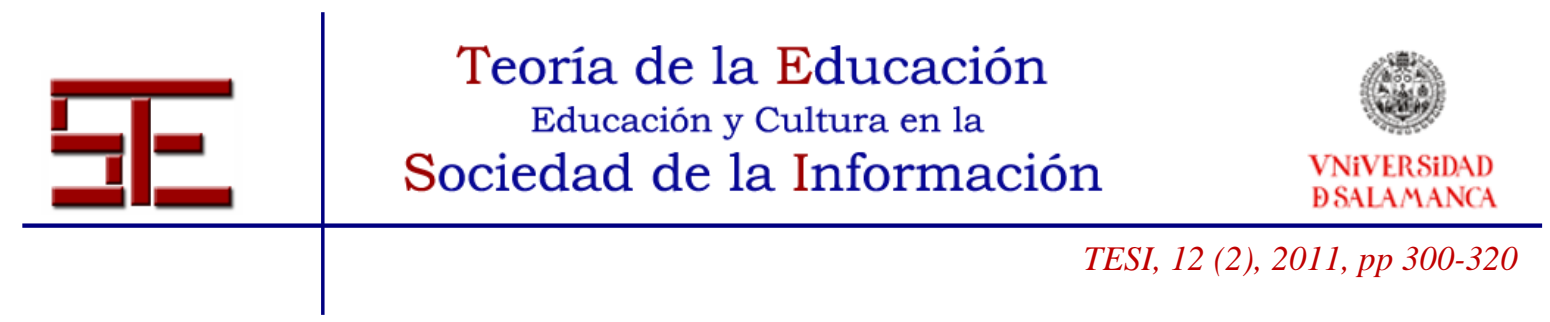

Para responder a este problema de investigación se estableció el siguiente objetivo general:

- Profundizar en las experiencias coeducativas del profesorado de EF y comprobar la relación con los contenidos del currículum de la materia.

Para poder alcanzar este objetivo con profundidad se establecieron objetivos secundarios con los que se pudiera analizar con más detalle la actuación docente:

a) Conocer las vivencias coeducativas del profesorado en relación con los contenidos de EF.

b) Analizar las diferentes estrategias metodológicas que se utilizan en cada bloque de contenidos.

Cabe indicar que el objeto del estudio excluye el análisis de las competencias básicas ya que, debido a su importancia, deben ser analizadas pormenorizadamente en una investigación independiente.

\section{MÉTODO}

\subsection{Participantes}

La población que participó en el estudio estuvo formada por 5 profesores (licenciados en EF o CAFD) de EF de Educación Secundaria Obligatoria. Los centros escolares donde desarrollan su labor docente son de titularidad pública o concertada. El muestreo que se llevó a cabo fue no probabilístico de casos típicos (Heinemann, 2008), siendo la experiencia menor a diez años un factor de exclusión. Dentro de un enfoque descriptivo e interpretativo, en este estudio se optó por la búsqueda de la calidad de la información derivada de las entrevistas, de tal manera que no se buscó la generalización de los resultados (Ruiz; Camps, 2009).

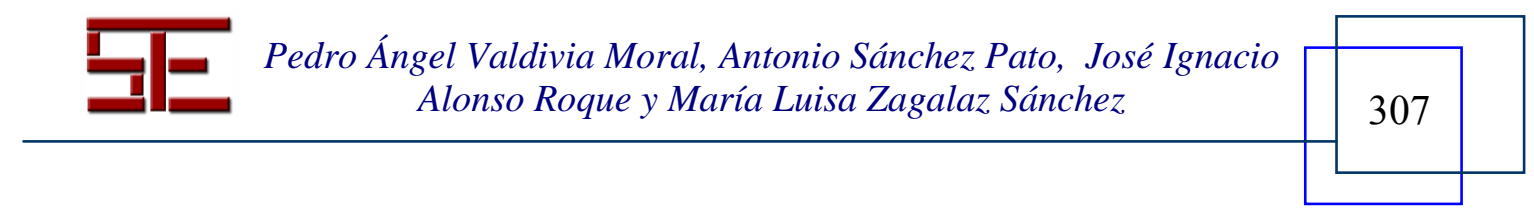




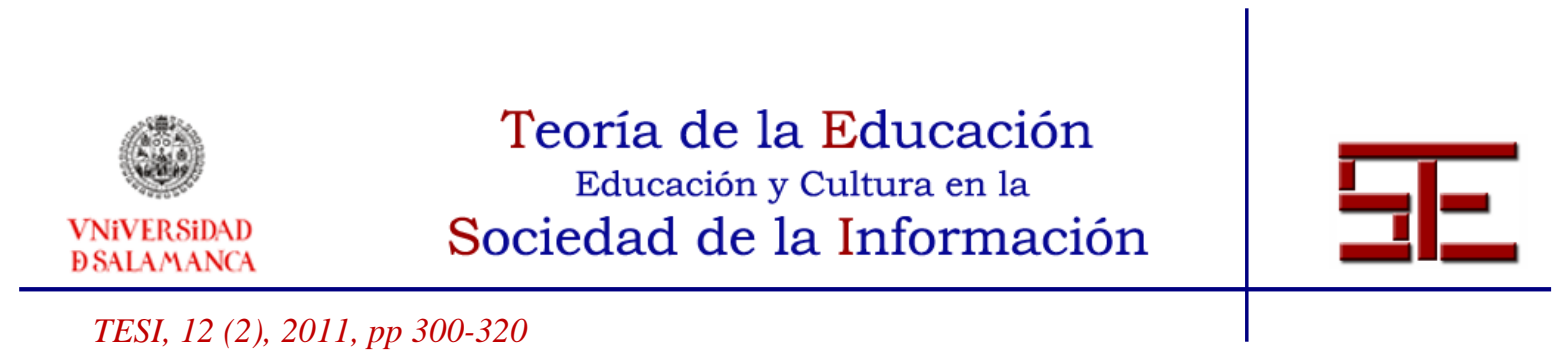

Tabla 1. Características de los participantes.

\begin{tabular}{ccccccc}
\hline Sujeto & Sexo & $\begin{array}{c}\text { Años en } \\
\text { Primaria }\end{array}$ & $\begin{array}{c}\text { Años en } \\
\text { Secundaria }\end{array}$ & $\begin{array}{c}\text { Titularidad } \\
\text { del centro }\end{array}$ & $\begin{array}{c}\text { Carácter } \\
\text { de los } \\
\text { centros }\end{array}$ & $\begin{array}{c}\text { Puestos } \\
\text { Específicos }\end{array}$ \\
\hline P1 & F & - & 27 & Pub & Mx & 2 años TAFAD \\
\hline P2 & M & 14 & 12 & Pub & Mx & - \\
\hline P3 & F & - & 30 & $\begin{array}{c}\text { Pub y Priv (9 } \\
\text { años })\end{array}$ & $\begin{array}{c}\text { Mx y Seg } \\
(9 \text { años })\end{array}$ & - \\
\hline P4 & M & & Pub & Mx & $\begin{array}{c}\text { Director del } \\
\text { centro }\end{array}$ \\
\hline P5 & M & 3 & 7 & Priv & Mx & - \\
\hline $\begin{array}{l}\text { Nota: Sexo: (M) Masculino, (F) Femenino. Titularidad del centro: (Pub) Publico, (Priv) Privado. Carácter de } \\
\text { los centros: (Mx) Mixto, (Seg) Segregado. Módulo de Grado superior de Técnico en Actividades Físicas y } \\
\text { Animación Deportiva (TAFAD). }\end{array}$ \\
\hline
\end{tabular}

\subsection{Procedimiento}

El primer paso fue la elaboración del marco teórico, donde se realizó una profunda revisión de la literatura científica sobre la temática. De esta manera, se estableció el estado de la cuestión y la necesidad de abordar un estudio.

La siguiente fase constó de tres etapas. En primer lugar, se concretaron los objetivos de la investigación, delimitándose la información útil para la elaboración del guion de las entrevistas. La segunda etapa se centró en la formación y el entrenamiento del entrevistador utilizando la propuesta de Anguera (2003). De esta manera, se adiestró al investigador mediante la realización de un seminario sobre técnicas de "role playing", para asimilar las cualidades necesarias para abordar las entrevistas. En la tercera etapa se abordó la elaboración y validación de la entrevista. Se utilizaron las pautas de Campoy (2006) para el diseño del guion: a) producción de preguntas de tipo semiestructurado y abierto para un enfoque cualitativo; b) realización de un estudio piloto mediante entrevistas a dos licenciados en CAFD; c) consulta a jueces expertos en la materia para dotar al instrumento de validez interna. Los jueces expertos eran

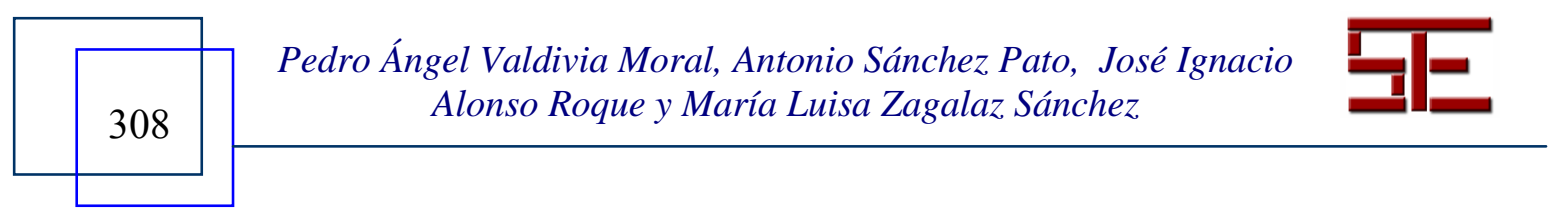




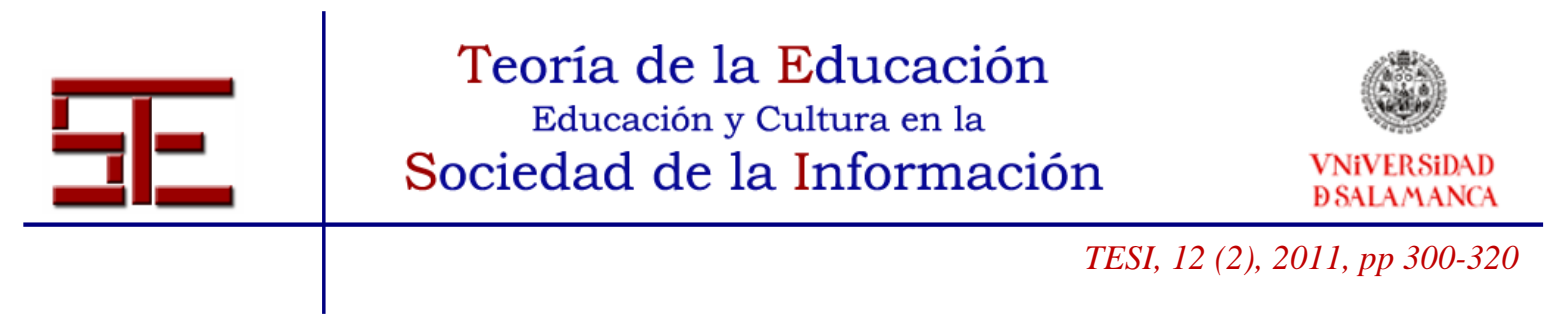

doctores en CAFD, Filosofía y Pedagogía. Con estos procesos se buscaron la corrección de los sesgos y la obtención del guion definitivo.

En la última fase el objetivo fue contactar con la población de estudio para concertar una reunión donde se les aportó información sobre el estudio y se fijaron las fechas para las entrevistas. Además se obtuvo un consentimiento informado del profesorado. Efectuadas las entrevistas, se recogieron los datos cualitativos procedentes de la transcripción de los discursos del profesorado.

\subsection{Instrumento y materiales}

El diseño de la entrevista semiestructurada estuvo caracterizado por el seguimiento de un guion por parte del entrevistador, de esta manera el modo de formular las preguntas queda a la valoración del entrevistador (Corbetta, 2007). Para poder obtener un enfoque cualitativo se profundizó en las experiencias del profesorado (ÁlvarezGayou, 2006).

Se utilizaron las siguientes estrategias para la obtención de la fiabilidad. Durante el proceso de análisis de contenido de los datos, para la fiabilidad externa se tuvieron en cuenta los problemas que describen Thomas y Nelson (2007):

1) Estatus del investigador.

2) Selección de los participantes.

3) Situación y condiciones sociales.

4) Métodos de recogida y análisis de los datos.

Para la fiabilidad interna se utilizaron las pautas de Goetz y LeCompte (1984, citados en Thomas y Nelson, 2007), que indican las siguientes estrategias: A) Descriptores de baja inferencia, mediante la transcripción literal de los discursos y apuntes de campo, incidiendo en emociones y otros puntos de la entrevista para aportar significado a los resultados. B) Examen por pares, para ello se consultó a investigadores afines a esta metodología de investigación. C) Datos registrados mecánicamente, utilizando dos grabadores digitales.

La triangulación de datos también fue una técnica utilizada para aportar fiabilidad al instrumento (Valles, 2007), consultando diversas fuentes documentales. Además se recurrió a la triangulación de investigadores, consultando a investigadores de diversas

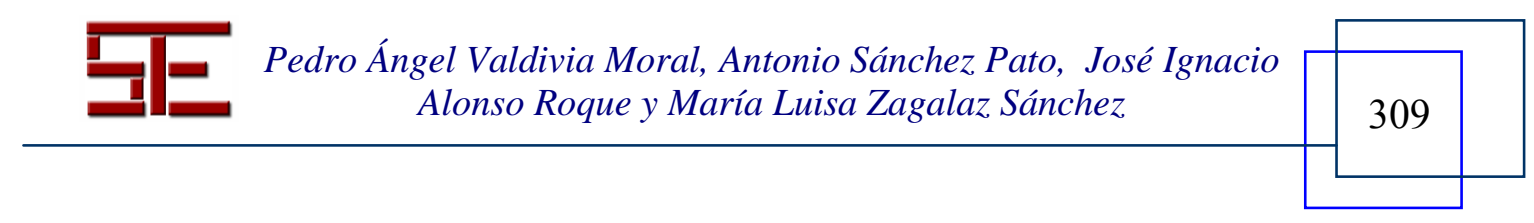




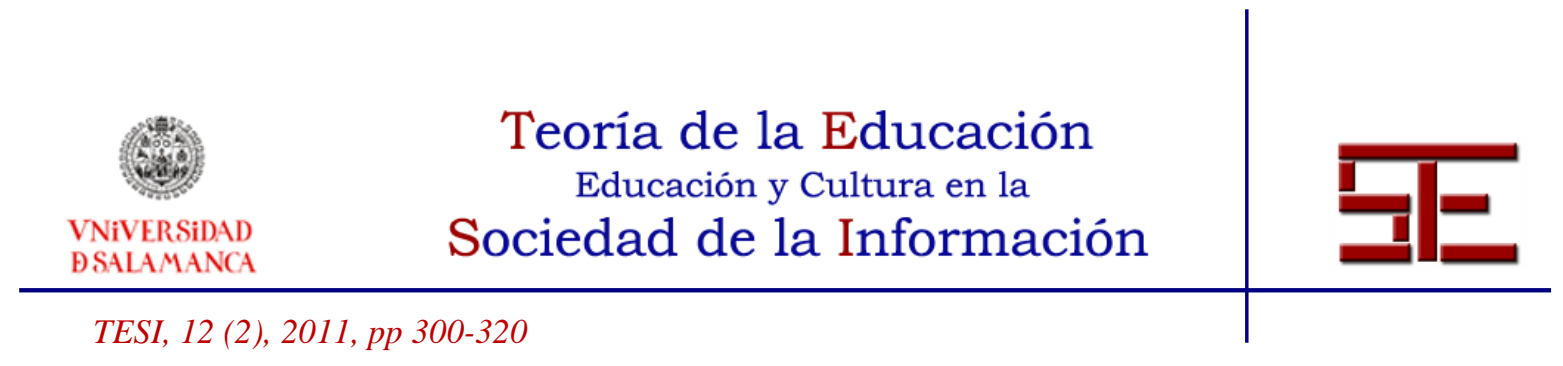

disciplinas. La entrevista final tenía una duración que varía entre 60 minutos y 80, en función del entrevistado.

\subsection{Análisis de contenido}

Se sometieron al análisis de contenido los textos transcritos de las entrevistas para desvelar el sentido del discurso (Pereira, 2005). De esta manera, se utilizó el modelo de análisis cualitativo de textos adaptados de Heinemann (2008), el cual consistía en:

1) Identificación de los objetos de estudio.

2) Elaboración de un mapa de categorías.

3) Subcategorías e indicadores.

4) Análisis de los textos.

5) Codificación de los textos.

6) Interpretación.

En primer lugar se seleccionaron y organizaron los datos en función del sistema de categorías a priori, para posteriormente realizar el análisis de los datos. De esta manera, algunas de las preguntas realizadas dieron lugar a la creación de subcategorías. Las categorías para el análisis de datos fueron (Tabla 2).

Tabla 2. Relación de categorías

A) Bloque de contenidos de "Condición Física y Salud".

B) Bloque de contenidos "Expresión Corporal".

C) Bloque de contenidos de "Juegos y Deportes".

D) Bloque de contenidos de "Actividades en el Medio Natural".

\section{RESULTADOS Y DISCUSIÓN}

En primer lugar, se debe destacar que no se han encontrado otros estudios que analicen los bloques de contenidos en EF desde un enfoque coeducativo desde la perspectiva del profesorado. Por ello, no podemos comparar los resultados con estudios previos.

Desde un enfoque general, se "cree que en los primeros cursos de secundaria, donde se pueden trabajar ciertas habilidades, no se muestran las diferencias, más aún, en estos cursos donde las diferencias en las capacidades físicas no son tan acentuadas" ${ }^{(\# 2)[1]}$.

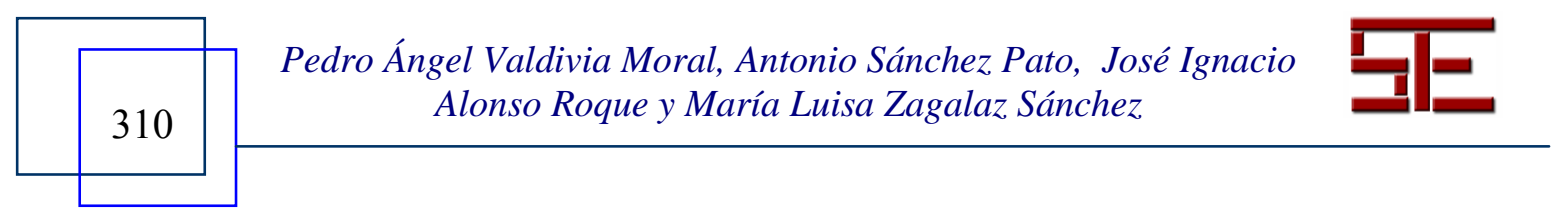




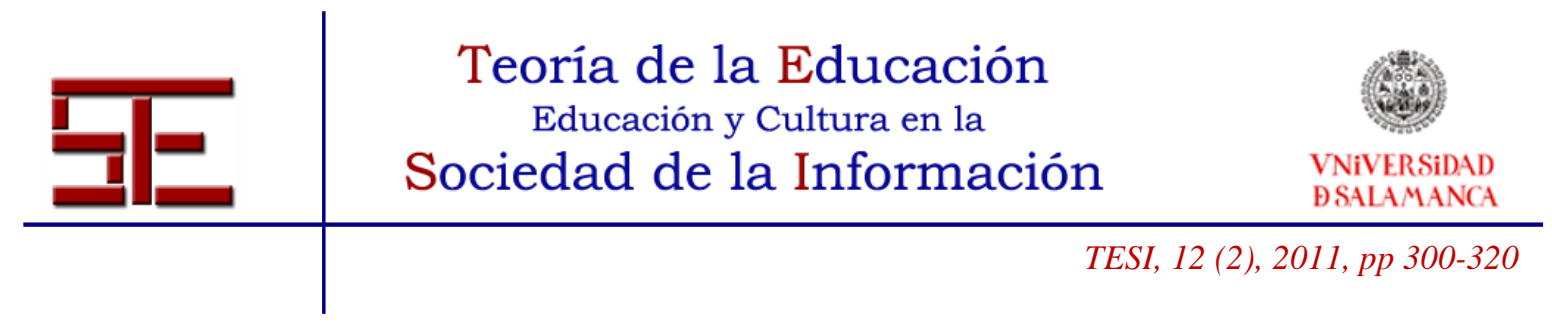

La opinión general es que "la coeducación debe ser trabajada en todos los bloques de contenidos. No debe ir relacionada a los bloques, sino a los objetivos que se marque cada docente" $"(\# 5)$.

\section{a) Categoría Bloque de contenidos de Condición Física y Salud}

En esta categoría se analizan los aspectos metodológicos de estos contenidos y su relación con la coeducación, destacando la percepción del profesorado sobre las diferencias fisiológicas entre los sexos y su repercusión en las clases.

Respecto al trabajo de estos contenidos de forma mixta se afirma que "cuando se abordan contenidos de Condición Física y Salud e intento la consecución de objetivos actitudinales como la cohesión de grupo, realizo tareas donde se tienen que ver implicados todos de manera conjunta" ${ }^{(\# 5)}$. Existen docentes que utilizan las diferencias fisiológicas de ambos sexos para abordar estos contenidos, de tal manera que un docente indica que "la Condición Física y Salud es un contenido que tradicionalmente ha estado relacionado con el sexo masculino. En mis clases busco que las ventajas fisiológicas de cada sexo como son la resistencia y la fuerza en hombres y la flexibilidad en mujeres, sirvan para desarrollar las actividades, por ejemplo, para trabajar la resistencia realizo carreras por parejas mixtas o por grupos mixtos, de tal manera que no resalten las diferencias individuales, sino el trabajo cooperativo" ${ }^{(\# 4)}$.

No obstante también existe la tendencia de trabajar estos contenidos de forma segregada de tal manera que "si realizamos pruebas físicas como la batería eurofit estamos obligados a separar por sexos" ${ }^{(\# 1)}$. Así mismo, desde un punto de vista de rendimiento físico se opina que "si se pretende dar a conocer al alumno cuales son sus parámetros de rendimiento físico y que realicen un ajuste más exhaustivo de la condición física, como por ejemplo puede ser el entrenamiento por intervalos, puede que tengan que agruparse por capacidad, coincidiendo de esta manera un mayor grupo de varones en el grupo de mayor nivel" ${ }^{(\# 5)}$.

\section{b) Categoría Bloque de contenidos de Expresión Corporal}

La mayoría de los docentes indican que es un bloque de contenidos complicado desde el punto de vista actitudinal, "si tengo la ocasión de trabajar con un grupo varios años, se observan diferentes etapas. Los chicos siempre son reticentes a estas actividades, independientemente de los grupos con los que trabajen. En los últimos

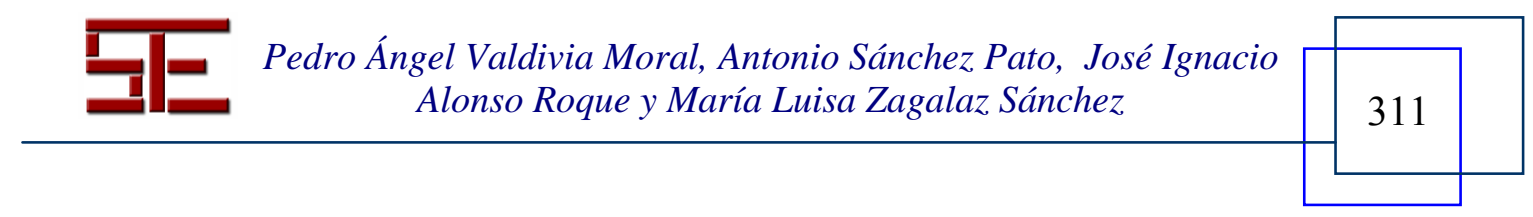




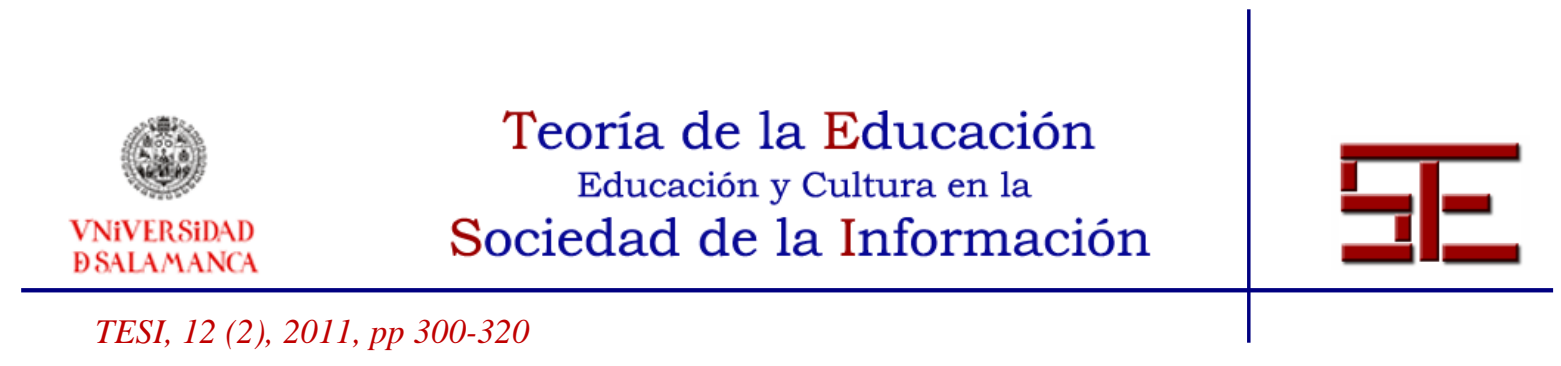

cursos, también las chicas se oponen en ciertos casos a trabajar en grupos donde se encuentre algún chico por le que se sientan atraídas, sin embargo, pierden la vergüenza con otros compañeros" (\#3). Una de las principales dificultades de estos contenidos reside en el propio profesorado ya que como reflexiona un docente, "mucho profesorado no aborda estos contenidos porque los desconoce, no se han formado en ellos o no se sienten atraídos y como existe poco control del currículum de EF, sus alumnos se quedan sin este tipo de formación de tal manera que cuando otro docente pretende trabajar estos contenidos con el grupo, se encuentra una oposición" ${ }^{(\# 3)}$. Esta afirmación sigue la línea de Vizuete (2002) que indica que durante el proceso de formación del docente, predominan los contenidos deportivos. Desde el alumnado también se encuentran dificultades, de tal manera que "a veces nos encontramos con alumnos que se oponen a trabajar estos contenidos porque los asocian con actitudes homosexuales. Esto es así porque en algunas ocasiones, desde la familia se refuerza la idea de que la EF es deporte. En estos casos debes ser coherente con unos criterios de evaluación y tomar las medidas que correspondan, creándose de esta manera una situación conflictiva" ${ }^{(\# 5)}$.

Dentro de las ventajas de este bloque de contenidos se indica que "este bloque es favorable para trabajarlo de forma mixta y buscar un fomento de las relaciones entre ellos" (\#1). Otro docente indica que "es necesario hacer participe al alumnado de las ventajas de este tipo de contenidos. Dentro de mis clases intento realizar bailes modernos o sevillanas cuando se acercan algunas fiestas, dándole de esta forma sentido a estos contenidos más allá del ámbito educativo para que también lo utilicen en su tiempo libre" ${ }^{(\# 2)}$. Otro docente nos indica que se ayuda de diferentes acontecimientos para poder abordar este contenido indicando que "me ayudo de fechas de entregas de premios cinematográficos para abordar temas de interpretación o el día de la Región para los bailes regionales" ${ }^{(\# 4)}$.

\section{c) Categoría Bloque de contenidos de Juegos y Deportes}

La actitud general del profesorado es que en primer lugar "se debe luchar contra los estereotipos que se transmiten desde actividades como el fútbol, la gimnasia rítmica o determinados juegos tradicionales" $(\# 1, \# 3, \# 4, \# 5)$.

Los contenidos de este bloque tienen gran aceptación entre el alumnado, así mismo, el profesorado indica que "los juegos y deportes son muy propicios para trabajar de forma coeducativa porque hay mucha comunicación" ${ }^{(\# 1)}$. Desde diferentes deportes se

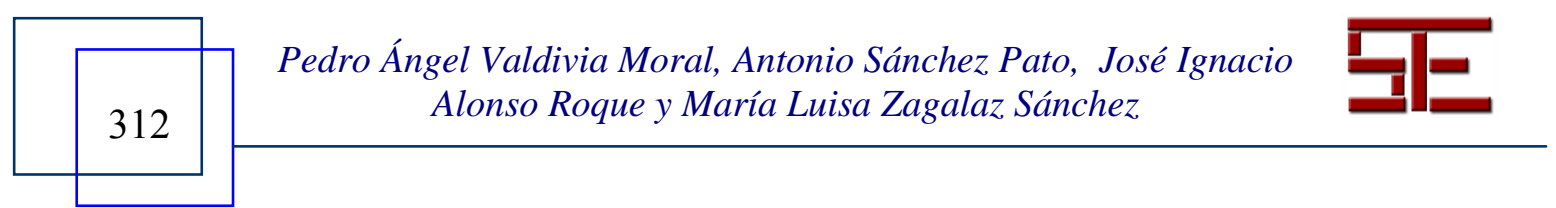




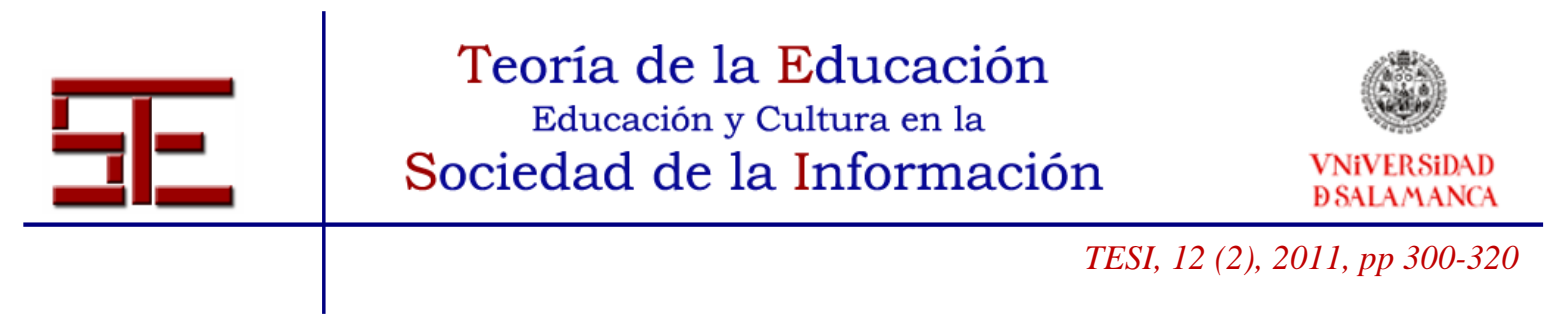

incide en el trabajo conjunto de ambos sexos, en esta línea un profesor opina que "tenemos deportes alternativos como el balonkorf o el kinball que por sus reglas obligan a que trabajen chicos y chicas, sin embargo, creo que desde el profesorado debemos convertir la participación conjunta en una actitud normalizada, no en una regla. De esta manera, en un deporte tan estereotipado como el fútbol debemos buscar la normalización de una práctica conjunta en nuestras clases" ${ }^{(\# 2)}$.

Existen deportes y juegos que, por sus características, los docentes los trabajan de forma distinta. Como ejemplo encontramos el rugby, en el que un docente indica que "aplico normas para evitar situaciones de impacto físico o agresivas, por ejemplo, para placar a un compañero solamente deben tocarlo con las dos manos en la cintura, pero en ningún caso abrazarlo para evitar de esta forma las caídas" ${ }^{(\# 1)}$. En otro sentido, otro profesor indica que "cuando practicamos rugby, tengo claro por mi experiencia que hay que separarlos porque ya han sido varias las ocasiones donde se han producido lesiones de consideración" ${ }^{(\# 3)}$.

Por último, uno de los docentes relaciona las actitudes de cada género con las actividades indicando que, "actualmente no se utilizan aparatos como el plinto o el potro. He sido entrenadora de gimnasia rítmica y el $80 \%$ de los chicos realizaba los juegos con estos aparatos sin dificultad, sin embargo, a las chicas les influía más el miedo y tenían más dificultades" ${ }^{(\# 3)}$.

\section{d) Categoría Bloque de contenidos de Actividades en el Medio Natural}

El profesorado no indica que existan dificultades significativas en este bloque de contenidos, sin embargo, se desprenden las siguientes opiniones. "En estos contenidos se identifica la orientación y algunas actividades relacionadas con el género masculino ya que tradicionalmente se ha tratado como una actividad de supervivencia. Quizás el profesorado hemos contribuido algo a esto, ya que en mis tiempos como estudiante de EF se trataba así este contenido en la facultad" (\#3). Otro docente indica que "cuando tratamos temas como la conservación del medio natural, las chicas son más sensibles a este tema" $(\# 5)$.

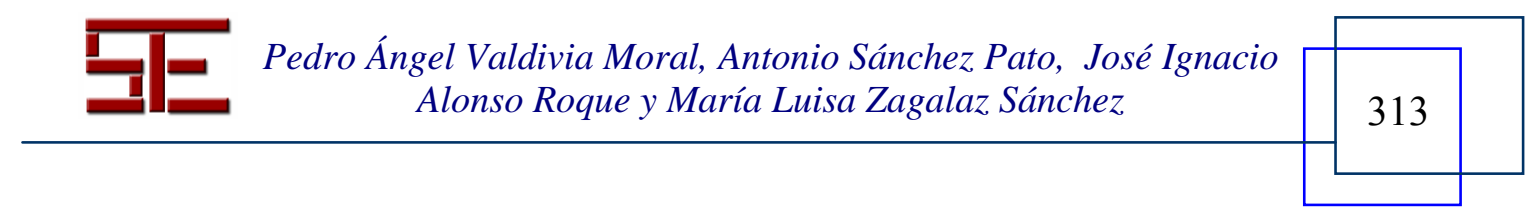




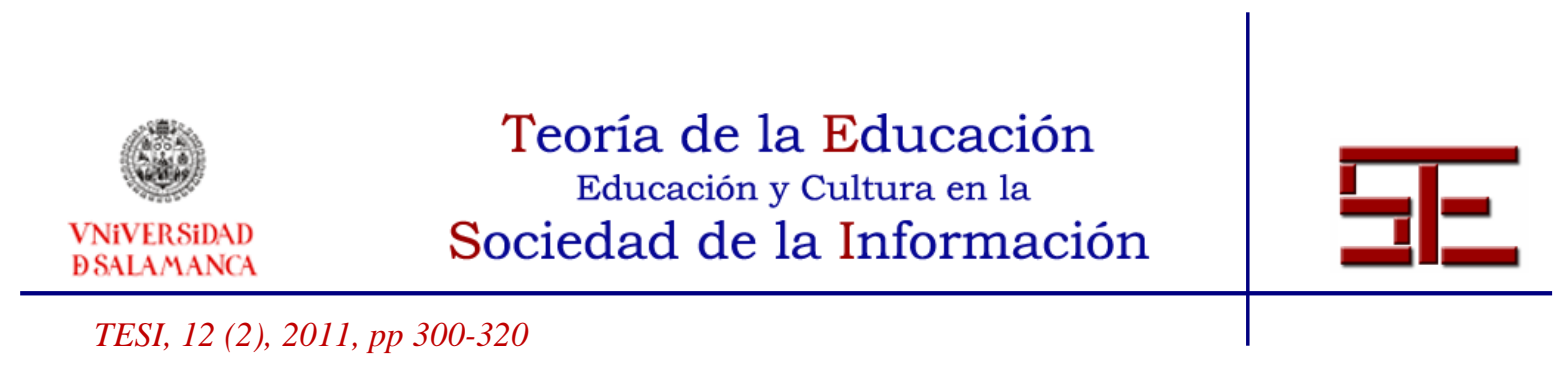

\section{CONTRIBUCIONES DEL ESTUDIO Y PROPUESTAS PEDAGÓGICAS}

Las categorías analizadas en este trabajo son reflejo de la relación de la coeducación con el trabajo de los contenidos de EF promulgados en el R.D. 1631/2006. A partir de aquí se hace necesaria una investigación descriptiva en la que se deberá diseñar y validar un cuestionario que se adapte a las demandas de un estudio sobre coeducación en EF; con ello, se comprobará si las vivencias y experiencias extraídas de este estudio son extrapolables a otros profesores de EF.

Además, es necesario comprobar si las técnicas coeducativas que el profesorado de EF pretende impartir tienen reflejo en el alumnado. De esta manera, se podrá identificar si se está actuando correctamente o si es necesaria alguna modificación con la que se consigan los objetivos coeducativos que el profesorado se proponga.

El bloque de contenidos de "Expresión Corporal" lleva añadidos problemas como: a) la oposición por parte de los alumnos a este contenido y b) el desconocimiento que parte del profesorado tiene sobre "Expresión Corporal". Por lo tanto, se hace necesaria la implantación de programas que formen al profesorado en las técnicas adecuadas para afrontar con éxito la consecución de objetivos de las diferentes temáticas que se pueden abordar en este bloque de contenidos.

Creemos que la coeducación es una parte importante en la formación de la persona y que el área de EF tiene un papel fundamental, ya que es el lugar donde se muestran más acusadamente las diferencias físicas entre sexos. Por lo tanto, el profesorado de EF debe incluir la coeducación dentro de sus objetivos para conseguir un aprendizaje que vaya más allá de los contenidos curriculares.

\section{CONCLUSIONES}

A través del análisis de las entrevistas interpretamos que las categorías creadas responden a los objetivos planteados respecto a la situación coeducativa actual en EF. Así, con la categoría "Bloque de contenidos de Condición Física y Salud" percibimos que el profesorado pretende que las diferencias fisiológicas por cuestión de sexo no se reproduzcan en las actividades y, caso de que estas diferencias se manifiesten, utilizan agrupaciones mixtas donde destaque el trabajo grupal. No obstante, cuando se busca el rendimiento, sí se realizan grupos de un solo sexo, ya sea intencionadamente por parte del profesor o por desarrollo normal de la actividad.

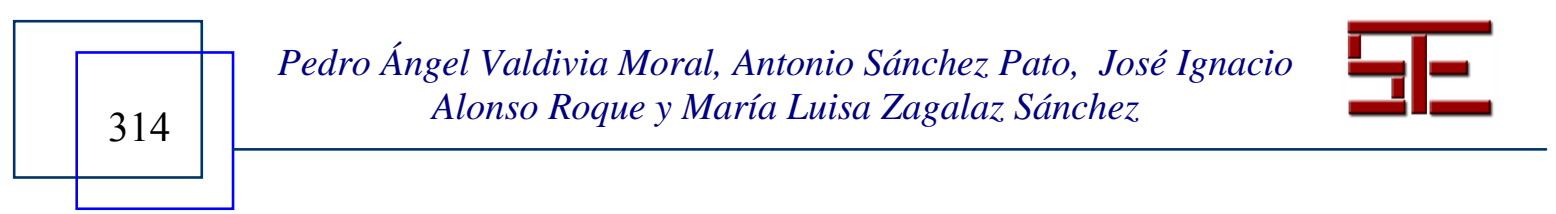




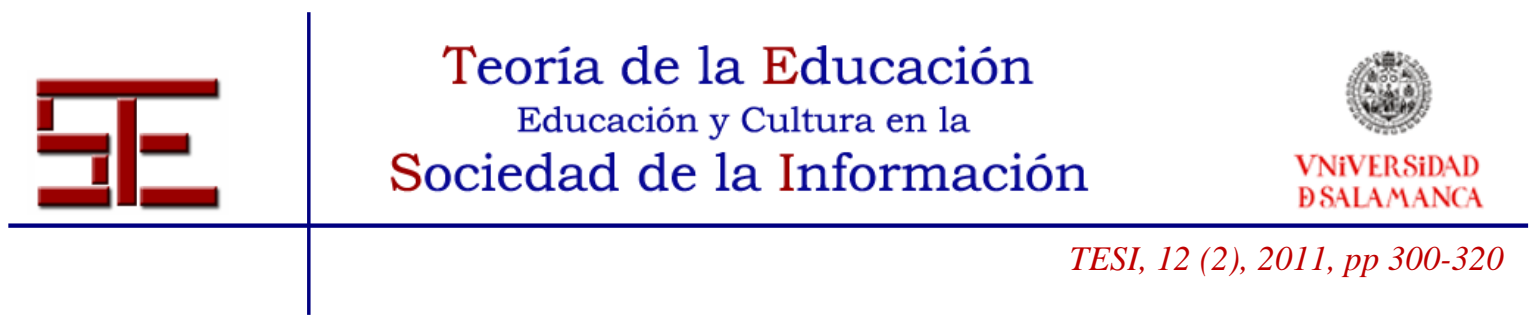

En virtud de la categoría "Bloque de contenidos de Expresión Corporal", se hace patente que existe profesorado que no aborda estos contenidos porque los desconoce. Por otro lado, se destaca que es un bloque de contenidos donde se encuentran dificultades actitudinales por parte de los chicos debido a los estereotipos preconcebidos por parte del alumnado. En cuanto a las estrategias metodológicas, el profesorado intenta dar significación a estos contenidos con el objetivo de que el alumnado perciba su funcionalidad.

Respecto a la categoría "Bloque de contenidos de Juegos y Deportes", el principal problema que encuentra el profesorado son los estereotipos que ciertos deportes llevan asociados. Respecto a las técnicas metodológicas en deportes con cierto nivel de peligrosidad no existe una opinión unánime, ya que en función del profesor se aboga por las actividades mixtas o segregadas en función del sexo.

Finalmente, sobre la categoría "Bloque de contenidos de Actividades en el Medio Natural", el profesorado considera que ciertas actividades estaban relacionadas con el sexo masculino cuando tomaban tintes de supervivencia. Actualmente, no encuentran barreras en ninguno de los dos sexos a la hora de trabajar estos contenidos.

\section{REFERENCIAS BIBLIOGRÁFICAS}

Álvarez-Gayou, J. (2006). Cómo hacer investigación cualitativa. Fundamentos y metodología. México D.F.: Paidós.

Anguera, M. T. (2003). La observación. En C. Moreno Rosset (Ed.), Evaluación psicológica. Concepto, proceso y aplicación en las áreas de desarrollo y de la inteligencia (pp. 271-308). Madrid: Sanz y Torres.

Azzarito, L. y Solmon, M. (2009). An Investigation of Students' Embodied Discourses in Physical Education: A Gender Project. Journal of Teaching in Physical Education, 28, 173-191.

Campoy Aranda, T. J. (2006). Técnicas cualitativas para la educación intercultural. En Pantoja Vallejo, A. y Campoy Aranda, T. J. (Eds.), Programas de intervención en educación intercultural (pp. 51-78). Granada: Grupo Editorial Interuniversitario.

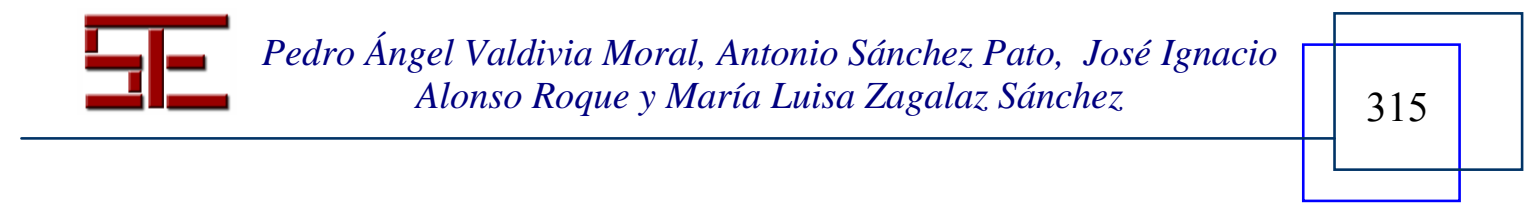




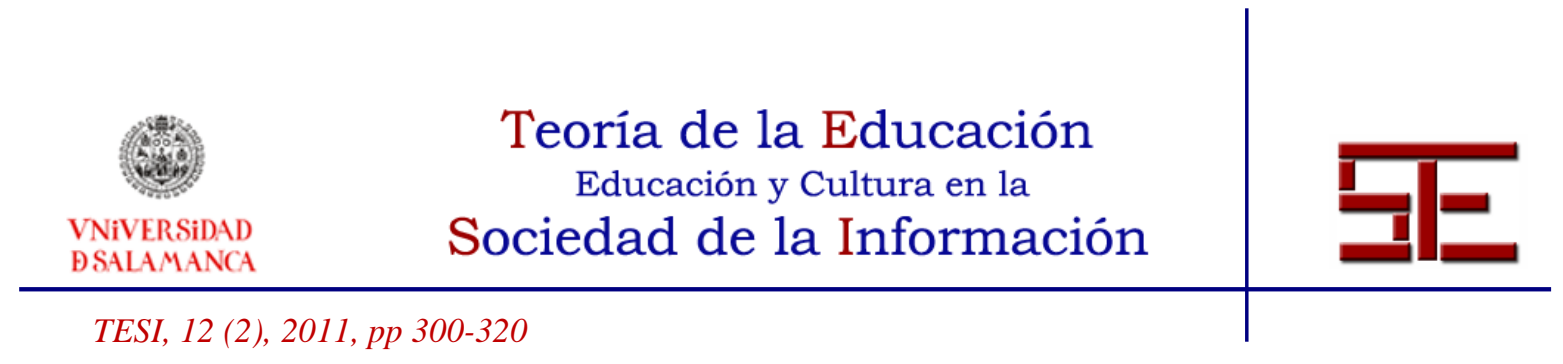

Castillo, M. A., Martínez-López, E. J. y Zagalaz, M. L. (2010). Analysis of the physical education teachers opinion from the Lakes Region (Chile) about the classes based on a mixed modality. Journal of Sport and Health Research, 2 (2), 77-94.

Corbetta, P. (2007). Metodología y técnicas de investigación social (Ed. Rev.). En La entrevista cualitativa (pp. 343-374). Madrid: Mc Graw Hill.

Fasting, K. (1989). Women's Lives - Consequences for Leisure and Sport. Nytt om Kvinneforskning, 13 (4), 18-26.

Fernández, E., Vázquez, B., Camacho, M. J., Sánchez Bañuelos, F., Martínez de Quel, O., Rodríguez, I., Rubia, A. y Aznar, S. (2006). La inclusión de la actividad física y el deporte en las mujeres adolescentes: estudio de los factores clave y pautas de intervención. En Vázquez, B. (Coord.), Las mujeres jóvenes y las actividades físicodeportivas (pp. 19-64). Madrid: Consejo Superior de Deportes.

Fontecha, M. (2006). Intervención didáctica desde la perspectiva de género en la formación inicial de un grupo de docentes en educación física. Tesis Doctoral. Universidad del País Vasco, Vitoria.

Freixas, A., García, E., Jiménez, J. R., Sánchez, J. L. y Santos, M. A. (1993). La coeducación, un compromiso social. Sevilla: Instituto Andaluz de la Mujer.

Gallo, C., González, C. y Salinero, J. J. (2010). Comparison of the motivaciones, perceptions and future expectations between physical activity and sport's sciencie students of Camilo José Cela (Spain) and Hertfordshire University (England). Journal of Sport and Health Research, 2 (3), 253-260.

Gómez, A., Díez, L. J., Fernández, J. M., Gorrín, A., Pacheco, J. J. y Sosa, G. (2008). Nueva propuesta curricular para el área de Educación Física en la Educación primaria. Revista Internacional de Medicina y Ciencias de la Actividad Física y el Deporte, 8 (29), 93-108.

González, M. (2005). ¿Tienen sexo los contenidos de Educación Física Escolar? Transmisión de estereotipos de sexo a través de los libros de texto en la etapa de secundaria. Revista Internacional de Medicina y Ciencias de la Actividad Física y del Deporte, 5 (18), 77-88.

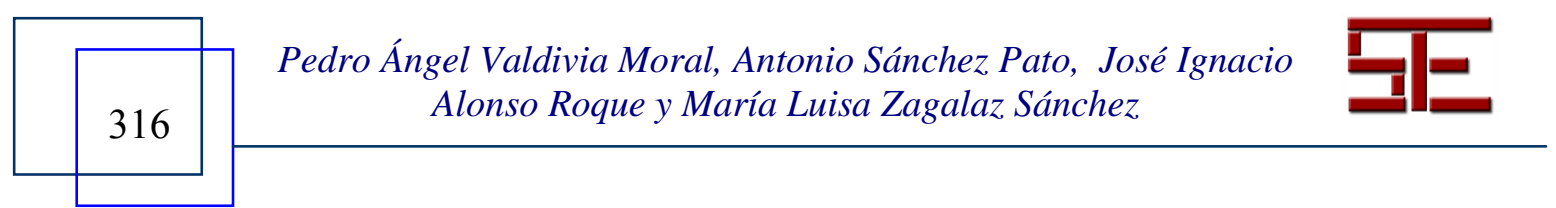




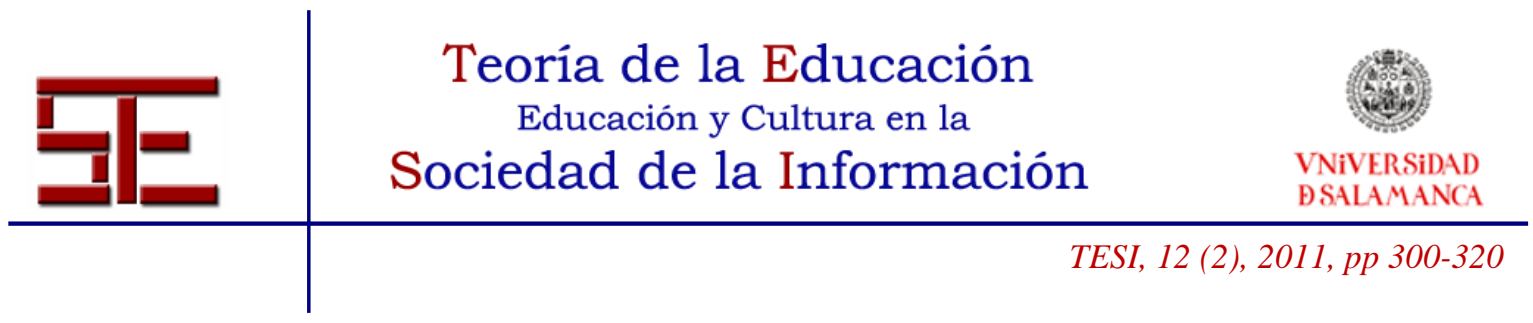

Gray, C. y Wilson, J. (2006). Teachers' experiences of a single-sex initiative in a coeducation school. Educational Studies, 32 (3), 285-298.

Heinemann, K. (2008). Introducción a la Metodología de la Investigación Empírica en las Ciencias del Deporte. Barcelona: Paidotribo.

Jiménez, R., Ramos, L.A., Cervelló, E., (2002). Análisis de la coeducación en las clases de Educación Física. Propuesta para una intervención no sexista en el contexto educativo. Habilidad motriz: Revista de Ciencias de la actividad física y del deporte, 18, 39-47.

Junta de Andalucía (2007). Ley 17/2007, de 10 de diciembre, de educación de Andalucía (LEA). B.O.J.A. no 252 de 26 de diciembre de 2007.

Koca, C. (2009). Gender interaction in coed physical education: a study in Turkey. Adolescence, 44 (173), pp. 165-185.

Martínez-Galindo, C. (2006). Motivación, coeducación y disciplina en estudiantes de Educación Física. Tesis Doctoral. Universidad de Murcia, Murcia.

Martínez Quesada, M. J. (2009). Coeducación: Una parte fundamental de la educación. Revista digital Transversalidad Educativa, 28, 120-129.

Ministerio de Educación y Ciencia (1990). Ley Orgánica 1/1990, de 3 de octubre, de Ordenación General del Sistema Educativo (LOGSE). B.O.E. no 238 de 4 de octubre de 1990.

Ministerio de Educación y Ciencia (2006). Ley Orgánica 2/2006, de 3 de mayo, de Educación (LOE). B.O.E. n ${ }^{\mathrm{o}} 136$, de 4 de mayo de 2006.

Ministerio de Educación y Ciencia (2006). Real Decreto 1631/2006, de 29 de diciembre, por el que se establecen las enseñanzas mínimas para la Educación Secundaria Obligatoria. B.O.E. $\mathrm{n}^{\mathrm{o}}$ 238, de 8 de diciembre de 2006.

Moreno, J.A., Alonso, N., Martínez Galindo, C. y Cervelló, E. (2005). Motivación, disciplina, coeducación y estado de flow en educación física: Diferencias según la

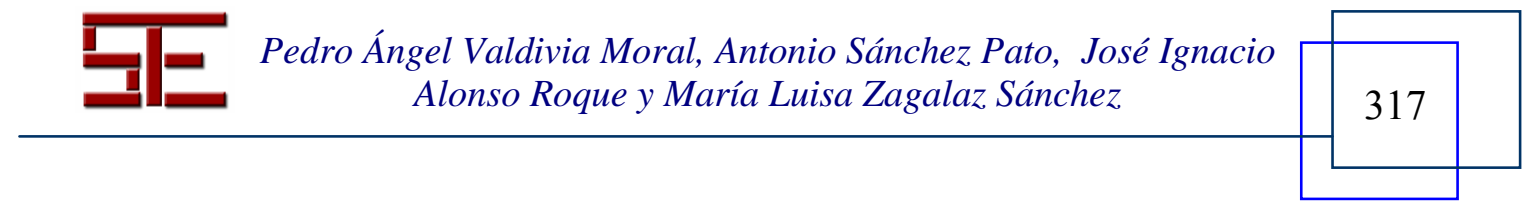




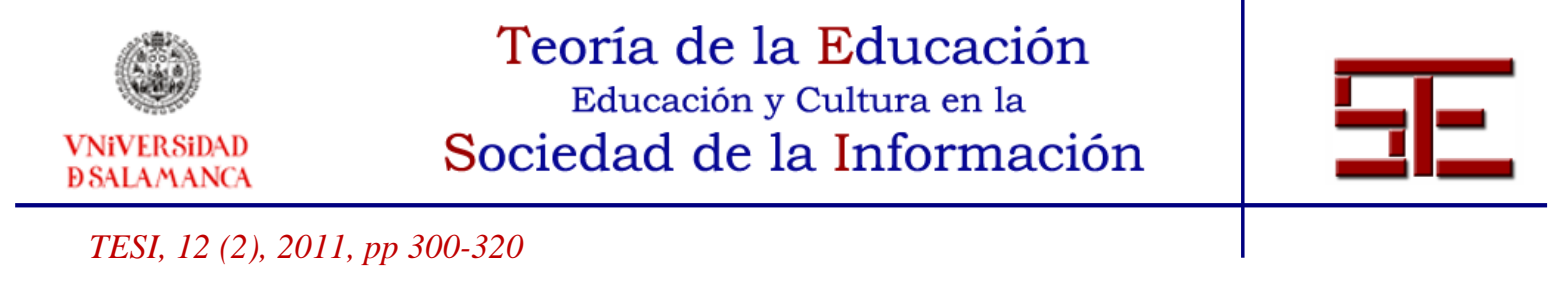

satisfacción, la práctica deportiva y la frecuencia de práctica. Cuadernos de psicología del deporte, 5, 1-2, 225-243.

Mosquera, Ma . J. y Puig, N. (1998). Género y edad en el deporte. En García Ferrando, M., Puig, N. y Lagardera, F. (Eds.), Sociología del deporte. Madrid: Alianza.

Mosquera, Ma . J., Sánchez, A. y Bada, J. (2004). No violencia en el deporte y en la visa. Guía para madres y padres. Xunta de Galicia: Dirección Xeral para o Deporte.

Nicaise, V., Cogerino, G., Fairclough, S., Bois, J. E. y David, K. L. (2007). Teacher feedback and interactions in physical education: Effects of student gender and physical activities. European review of physical education, 13, 319-337.

Pereira, A. L. (2005). Sentidos del alpinismo de elevada y extrema altitud: análisis efectuado a partir de los discursos de alpinistas portugueses y españoles. Cultura, Ciencia y Deporte, 2 (1), 57-64.

Pérez, I. y Carbó, J. R. (2010). Juegos de Rol y Roles de Género. Teoría de la Educación. Educación y Cultura en la Sociedad de la Información, 11 (3), 168-184.

Ramos, L. A. y Del Villar, F. (1999). Criterios de estructuración de contenidos para el área de Educación Física en la Enseñanza Secundaria Obligatoria. Apunts: Educación Física y Deportes, 56, 32-38.

Robles, J., Giménez, F. J. y Abad, M. T. (2010). Motivos que llevan a los profesores de Educación Física a elegir los contenidos deportivos en la E.S.O. Retos. Nuevas tendencias en Educación Física, deporte y recreación, 18, pp. 5-8.

Ruiz, U. y Camps, A. (2009). Investigar los géneros discursivos en el proceso educativo. Revista de Psicodidáctica, 14 (2), 211-228.

Salomone, R. (2009). Igualdad y diferencia. La cuestión de la equidad de género en la educación. Revista Española de Pedagogía, 65 (238), 433-446.

Soler, S. (2006). Actitudes y relaciones de niñas y niños ante contenidos de la educación física en primaria estereotipados por el género: el caso del fútbol. En Vázquez, B.

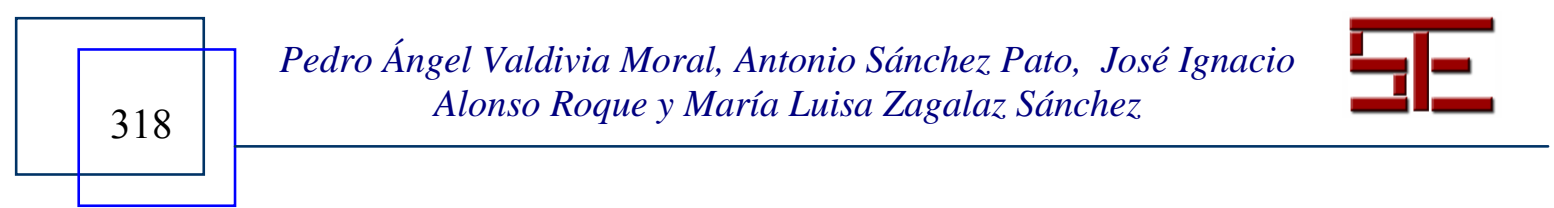




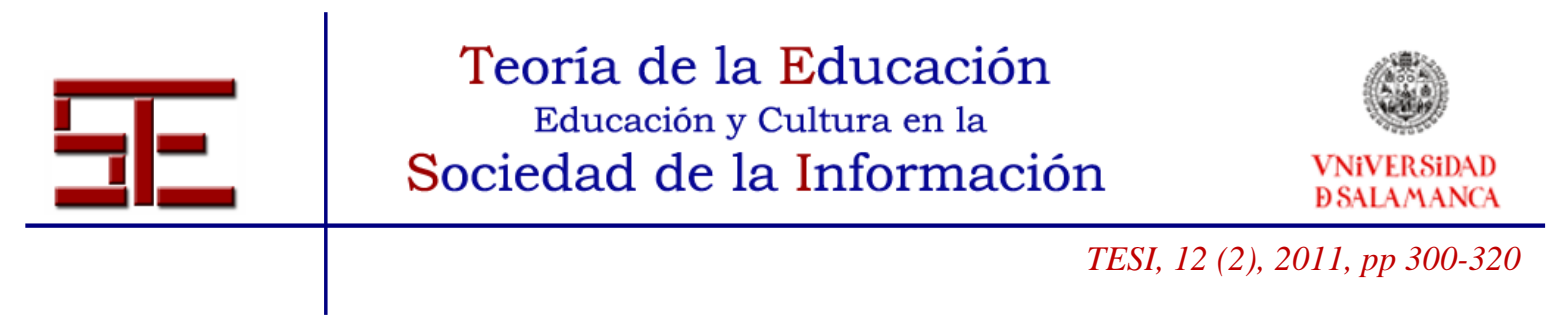

(Coord.), Las mujeres jóvenes y las actividades físico-deportivas (pp. 119-150). Madrid: Consejo Superior de Deportes.

Subirats, M. (1994). Conquistar la igualdad: La coeducación hoy. Revista iberoamericana de educación, 6, 49-78.

Subirats, M., Brullet, C., (1988). Rosa y azul. La transmisión de los géneros en la escuela mixta. Madrid: Instituto de la Mujer. Ministerio de Cultura.

Tainta, P. (2007). Una mirada hacia atrás (LOGSE) y un paso hacia delante (LOE) en la formación del profesorado de ESO. ESE: Estudios sobre Educación, 12, 139-166.

Thomas, J.R. y Nelson, J.K. (2007). Research methods in physical activity. Champaign, Il: Human Kinetics.

Valdivia, P. A. (2010). La coeducación en el sistema educativo español. Un análisis desde la Educación Física. Jaén: Asociación Didáctica Andalucía.

Valdivia, P. A., Sánchez, A., Alonso, J. I. y Zagalaz, M. L. (2010). La coeducación en el área de Educación Física en España: una reseña histórica. Cultura, Ciencia y Deporte, 5 (14), 77-83.

Valles, M. S. (2007). Técnicas cualitativas de investigación social. Diseños y estrategias metodológicas en los estudios cualitativos (pp. 69-108). Madrid: Edit. Síntesis.

Vázquez, B. (1990). La coeducación en Educación Física. Algunas reflexiones. En: Cursos Emakunde. Primer postgrado de coeducación. Hacia una escuela coeducadora (pp. 291-297). Álava: UPV.

Vázquez, B. y Alfaro, E. (2006). Presentación. En Vázquez, B. (Coord.), Las mujeres jóvenes y las actividades físico deportivas (pp. 9-18). Madrid: Consejo Superior de Deportes.

Vizuete, M. (2002). La didáctica de la educación física y el "área de conocimiento de expresión corporal: profesores y currículum”. Revista de Educación, 328, 137-154.

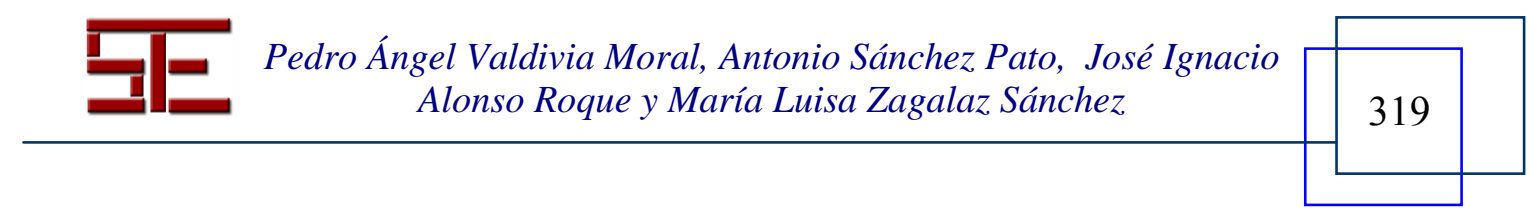




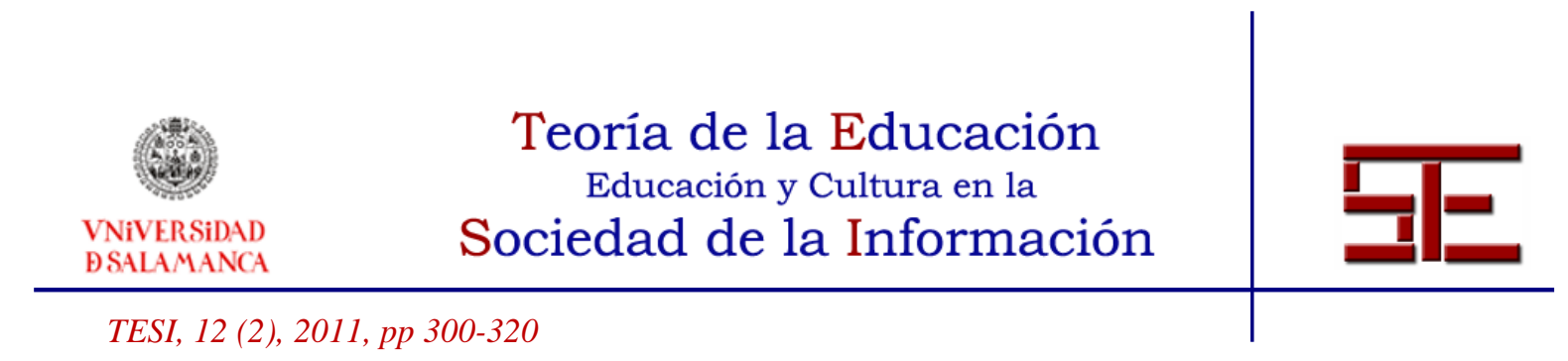

Zagalaz, M. L. (2001). Bases Teóricas de la Educación Física y del Deporte. Jaén: Universidad de Jaén.

Zagalaz, M. L., Arteaga, M., Cepero, M., Martos, M. M., Moreno, R., Rodrigo, M., (2000). Los temas transversales, interdisciplinariedad y currículum oculto en Educación Física. En Zagalaz, M. L. y Cepero, M. (Eds.), Educación Física y su Didáctica. Manual para el maestro generalista (pp. 293-318). Jaén: Jabalcuz.

\section{NOTAS}

[1] Los relatos del profesorado están numerados de acuerdo con el orden de realización de las entrevistas. Así, el entrevistado número 1 es identificado como \#1, y así sucesivamente.

Para citar el presente artículo puede utilizar la siguiente referencia:

Valdivia Moral, P. A., Sánchez Pato, A., Alonso Roque, J. I. y Zagalaz Sánchez, M. L. (2011). Experiencias coeducativas del profesorado de educación física y relación con los contenidos de la materia, en Barrios Vicente, I. M. (Coord.) Mujeres y la sociedad de la Información. Revista Teoría de la Educación: Educación y Cultura en la Sociedad de la Información. Vol. 12, n ${ }^{\circ}$ 2. Universidad de Salamanca, pp. 300-320 [Fecha de consulta: $\mathrm{dd} / \mathrm{mm} /$ aaaa].

http://campus.usal.es/ revistas_trabajo/index.php/revistatesi/article/view/8283/8287

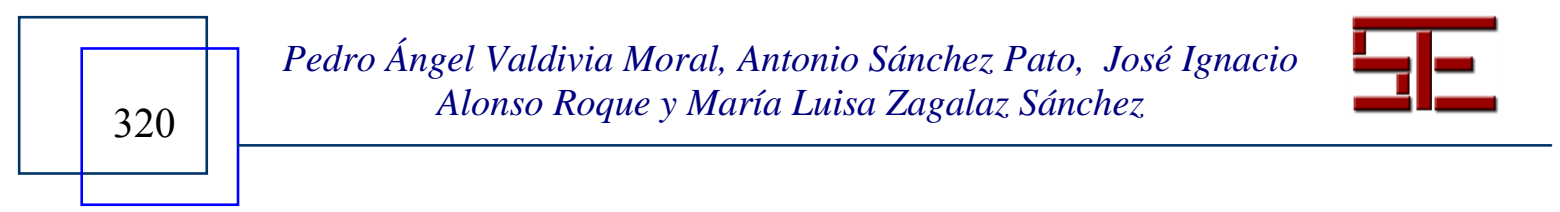

